\title{
Liquid-Liquid Phase Separation of Viologen Bistriflimide/Benzene mixtures: Role of the Dual Ionic and Organic Nature of Ionic Liquids
}

\author{
Shen $\mathrm{Li}^{1,2}$, Niloufar Safari ${ }^{3}$, Giacomo Saielli ${ }^{3,4 *}$, Yanting Wang ${ }^{1,2, *}$
}

${ }^{1}$ CAS Key Laboratory of Theoretical Physics, Institute of Theoretical Physics, Chinese Academy of Sciences, 55 East Zhongguancun Road, P. O. Box 2735, Beijing, 100190 China

${ }^{2}$ School of Physical Sciences, University of Chinese Academy of Sciences, 19A Yuquan Road, Beijing, 100049 China

${ }^{3}$ Department of Chemical Sciences, University of Padova, Via Marzolo, 1 - 35131, Padova, Italy.

${ }^{4}$ CNR Institute on Membrane Technology, Unit of Padova, Via Marzolo 1, 35131 Padova, Italy

\section{Supporting Information}

Table of sponge-like phase experimental mole ratios

p. S2

Table of NMR-DOSY diffusion coefficients

p. S3

Table of viologen and benzene chemical shifts

p. S3

Figure of viologen and benzene chemical shifts

p. S4

Figure of RDF

p. S4

Snapshots

p. S6

Coordination numbers of benzene around the viologen

p. S7

Synthesis and characterization

p. $\mathrm{S} 8$

${ }^{1} \mathrm{H}$ and ${ }^{13} \mathrm{C}$ NMR spectra

p. S9 
Table S1. Mole ratio $R=N_{\text {benzene }} / N_{\text {viologen }}$ in the sponge-like phase for the investigated systems in the phase diagram of Figure 3. V $(s)$ : solid viologen phase; B $(l)$ : liquid benzene phase; SP $(l)$ : liquid sponge-like phase. The $m+n$ represents the total length of two side chains of the cation.

\begin{tabular}{c|ccc}
\hline Viologen & $m+n$ & $R$ & Phase type \\
\hline $\mathrm{C}_{4} \mathrm{bpC}_{4}$ & 8 & - & $\mathrm{V}(s)+\mathrm{B}(l)$ \\
\hline $\mathrm{C}_{7} \mathrm{bpC}_{7}$ & 14 & 6.7 & $\mathrm{SP}(l)+\mathrm{B}(l)$ \\
$\mathrm{C}_{7} \mathrm{bpC}_{9}$ & 16 & 7.1 & $\mathrm{SP}(l)+\mathrm{B}(l)$ \\
$\mathrm{C}_{8} \mathrm{bpC}_{8}$ & 16 & 7.0 & $\mathrm{SP}(l)+\mathrm{B}(l)$ \\
$\mathrm{C}_{7} \mathrm{bpC}_{10}$ & 17 & 7.7 & $\mathrm{SP}(l)+\mathrm{B}(l)$ \\
$\mathrm{C}_{9} \mathrm{bpC}_{9}$ & 18 & 8.3 & $\mathrm{SP}(l)+\mathrm{B}(l)$ \\
$\mathrm{C}_{7} \mathrm{bpC}_{11}$ & 18 & 9.0 & $\mathrm{SP}(l)+\mathrm{B}(l)$ \\
$\mathrm{C}_{11} \mathrm{bpC}_{11}$ & 22 & 11.4 & $\mathrm{SP}(l)+\mathrm{B}(l)$ \\
$\mathrm{C}_{12} \mathrm{bpC}_{12}$ & 24 & 12.9 & $\mathrm{SP}(l)+\mathrm{B}(l)$ \\
$\mathrm{C}_{8} \mathrm{bpC}_{16}$ & 24 & 13.6 & $\mathrm{SP}(l)+\mathrm{B}(l)$ \\
$\mathrm{C}_{10} \mathrm{bpC}_{16}$ & 26 & 15.4 & $\mathrm{SP}(l)+\mathrm{B}(l)$ \\
$\mathrm{C}_{14} \mathrm{bpC}_{14}$ & 28 & 16.6 & $\mathrm{SP}(l)+\mathrm{B}(l)$ \\
$\mathrm{C}_{16} \mathrm{bpC}_{16}$ & 32 & 19.5 & $\mathrm{SP}(l)+\mathrm{B}(l)$ \\
\hline $\mathrm{C}_{7} \mathrm{bpC}_{7}$ & 14 & 1.1 & $\mathrm{SP}(l)+\mathrm{V}(s)$ \\
$\mathrm{C}_{9} \mathrm{bpC}_{9}$ & 18 & 2.2 & $\mathrm{SP}(l)+\mathrm{V}(s)$ \\
$\mathrm{C}_{14} \mathrm{bpC}_{14}$ & 24 & 5.0 & $\mathrm{SP}(l)+\mathrm{V}(s)$ \\
\hline $\mathrm{C}_{7} \mathrm{bpC}_{7}$ & 14 & 2.5 & $\mathrm{SP}(l)$ \\
$\mathrm{C}_{9} \mathrm{bpC}_{9}$ & 18 & 6.3 & $\mathrm{SP}(l)$ \\
$\mathrm{C}_{11} \mathrm{bpC}_{11}$ & 22 & 4.4 & $\mathrm{SP}(l)$ \\
$\mathrm{C}_{12} \mathrm{bpC}_{12}$ & 24 & 5.1 & $\mathrm{SP}(l)$ \\
\hline & & & \\
\hline
\end{tabular}


Table S2. Diffusion coefficients obtained by DOSY-NMR spectroscopy for the biphasic systems composed of liquid benzene, B $(l)$, in equilibrium with the liquid sponge-like phase, SP $(l)$, at $298 \mathrm{~K}$.

\begin{tabular}{c|ccc}
\hline Viologen & $m+n$ & $\begin{array}{c}D_{\text {benzene }} \\
10^{-5} \mathrm{~cm}^{2} / \mathrm{s}\end{array}$ & $\begin{array}{c}D_{\text {viologen }} \\
10^{-5} \mathrm{~cm}^{2} / \mathrm{s}\end{array}$ \\
\hline $\mathrm{C}_{8} \mathrm{bpC}_{8}$ & 16 & $3.79 \mathrm{E}-01$ & $1.98 \mathrm{E}-02$ \\
$\mathrm{C}_{9} \mathrm{bpC}_{9}$ & 18 & $4.43 \mathrm{E}-01$ & $1.78 \mathrm{E}-02$ \\
$\mathrm{C}_{7} \mathrm{bpC}_{11}$ & 18 & $4.54 \mathrm{E}-01$ & $1.97 \mathrm{E}-02$ \\
$\mathrm{C}_{11} \mathrm{bpC}_{11}$ & 22 & $6.31 \mathrm{E}-01$ & $1.88 \mathrm{E}-02$ \\
\hline $\mathrm{C}_{12} \mathrm{bpC}_{12}$ & 24 & $7.00 \mathrm{E}-01$ & $1.77 \mathrm{E}-02$ \\
$\mathrm{C}_{16} \mathrm{bp}_{8}$ & 24 & $7.10 \mathrm{E}-01$ & $2.10 \mathrm{E}-02$ \\
$\mathrm{C}_{14} \mathrm{bppC}_{14}$ & 28 & $8.95 \mathrm{E}-01$ & $2.80 \mathrm{E}-02$ \\
$\mathrm{C}_{16} \mathrm{bpC}_{16}$ & 32 & $1.06 \mathrm{E}+00$ & $4.06 \mathrm{E}-02$ \\
\hline
\end{tabular}

Table S3. Chemical shifts, in ppm, of viologen ring protons and benzene in the sponge-like phase, SP $(l)$, at $298 \mathrm{~K}$, along the coexistence line with the pure benzene solution.

\begin{tabular}{|c|c|c|c|c|c|}
\hline $\mathrm{n}$ & $\mathrm{m}$ & $n+m$ & $\begin{array}{l}\delta\left({ }^{1} \mathrm{H}\right) \\
\text { ortho }\end{array}$ & $\begin{array}{l}\delta\left({ }^{1} \mathrm{H}\right) \\
\text { meta }\end{array}$ & $\begin{array}{l}\delta\left({ }^{1} \mathrm{H}\right) \\
\text { benzene }\end{array}$ \\
\hline 7 & 7 & 14 & 8.2 & 7.77 & 7.07 \\
\hline 8 & 8 & 16 & 8.22 & 7.82 & 7.07 \\
\hline 7 & 9 & 16 & 8.21 & 7.79 & 7.07 \\
\hline 9 & 9 & 18 & 8.21 & 7.82 & 7.07 \\
\hline 7 & 11 & 18 & 8.24 & 7.83 & 7.07 \\
\hline 11 & 11 & 22 & 8.23 & 7.86 & 7.07 \\
\hline 16 & 8 & 24 & 8.24 & 7.88 & 7.07 \\
\hline 12 & 12 & 24 & 8.24 & 7.88 & 7.07 \\
\hline 14 & 14 & 28 & 8.25 & 7.91 & 7.07 \\
\hline 16 & 16 & 32 & 8.28 & 7.95 & 7.07 \\
\hline
\end{tabular}




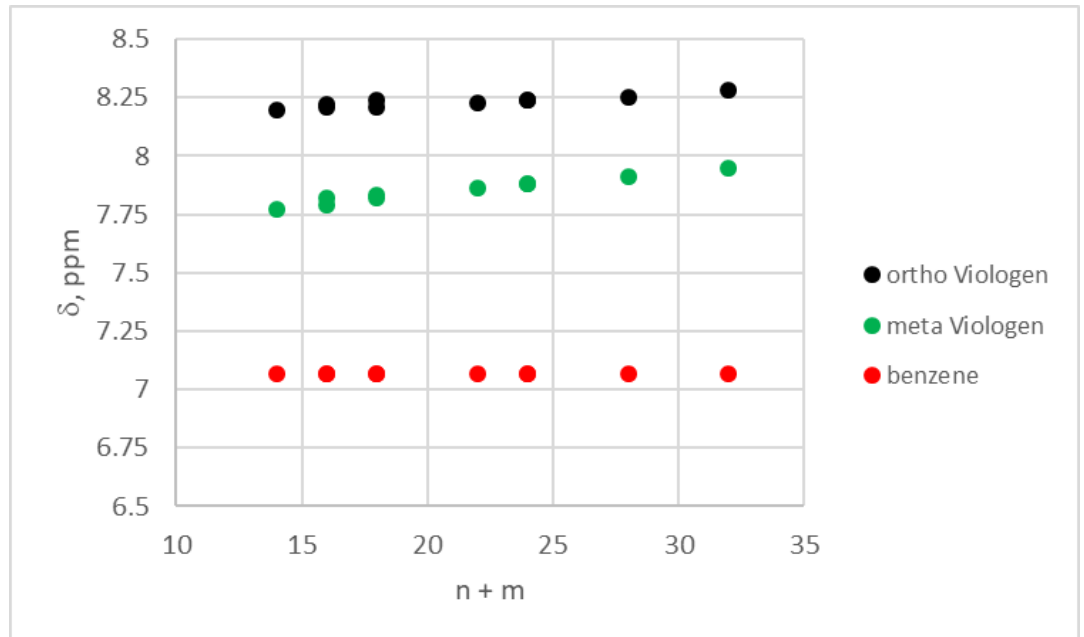

Figure S1. Chemical shifts, in ppm, of viologen ring protons and benzene in the sponge-like phase, SP $(l)$, at $298 \mathrm{~K}$, along the coexistence line with the pure benzene solution.
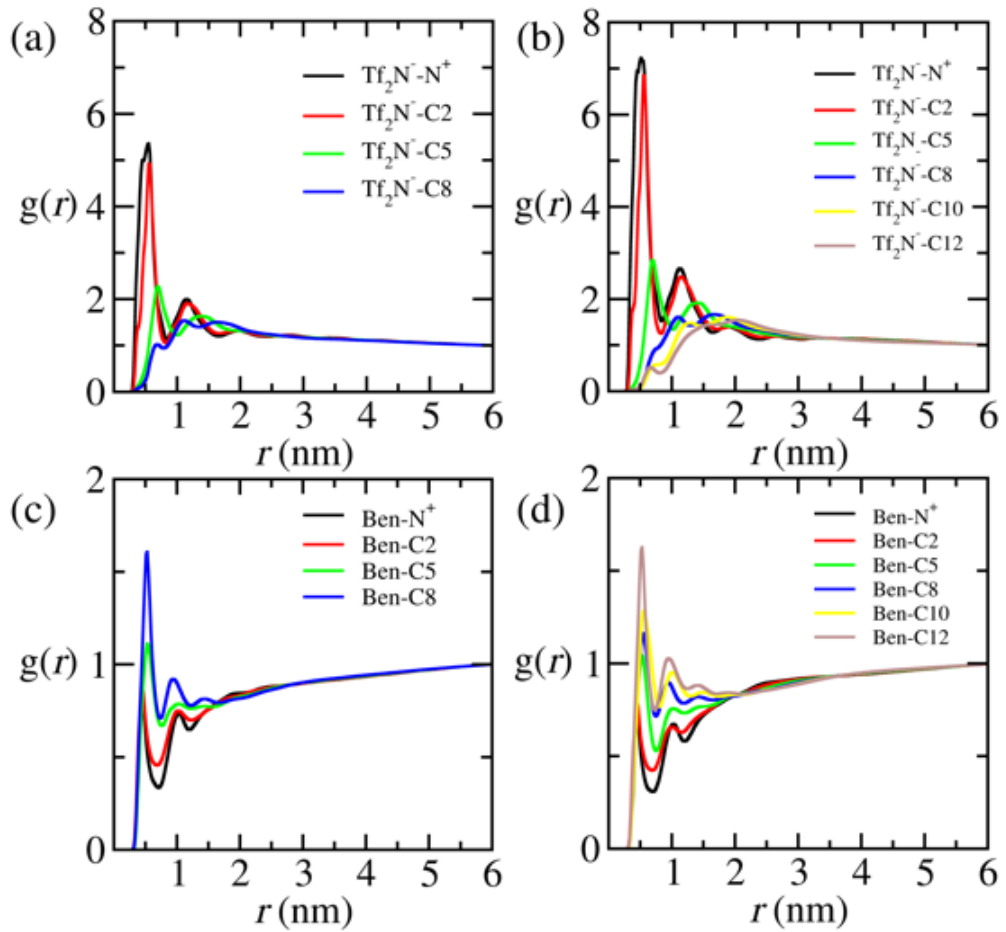

Figure S2. The RDFs between the geometric centers of anions and different atoms on $\mathrm{C}_{8} \mathrm{bpC}_{8}$ (a) and $\mathrm{C}_{12} \mathrm{bpC}_{12}(\mathrm{~b})$, and the RDFs between the geometric centers of benzene molecules (labelled as Ben) and different atoms on $\mathrm{C}_{8} \mathrm{bpC}_{8}(\mathrm{c})$ and $\mathrm{C}_{12} \mathrm{bpC}_{12}(\mathrm{~d}) . \mathrm{N}^{+}$represents the charged nitrogen on viologen cation and $\mathrm{C}_{x}(x=2,5,8,10,12)$ represent different carbon atoms on alkyl side chain away from $\mathrm{N}^{+}$with the distance of $x$. 

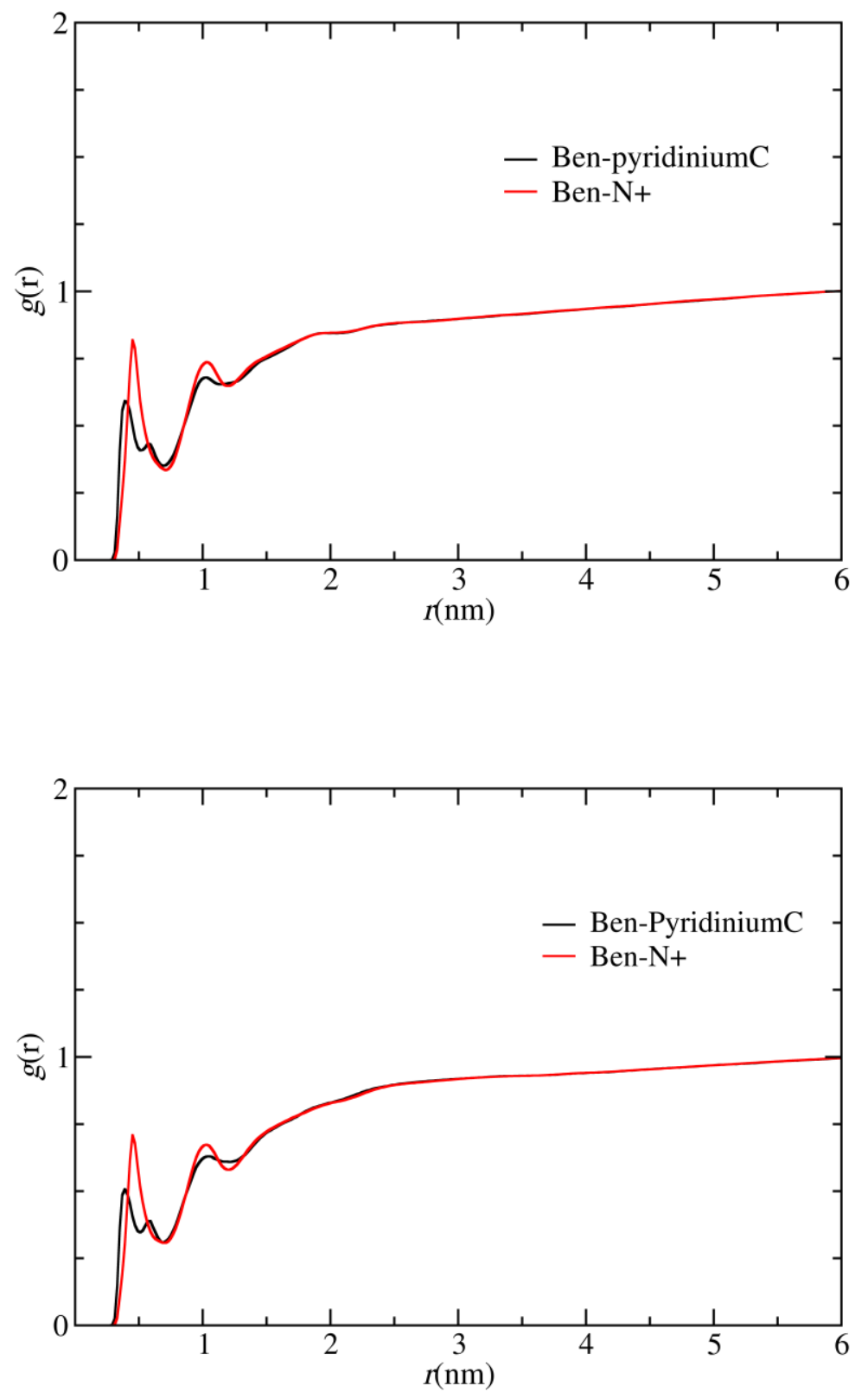

Figure S3: Comparison of the RDF between the center of mass of Benzene and pyridinium $\mathrm{N}^{+} / \mathrm{C}$ of (top) $\left[\mathrm{C}_{8} \mathrm{bpC}_{8}\right]^{2+}$ and (bottom) $\left[\mathrm{C}_{12} \mathrm{bpC}_{12}\right]^{2+}$. The black trace is the average of the various RDF of the $\mathrm{C}$ carbons of the ring. 

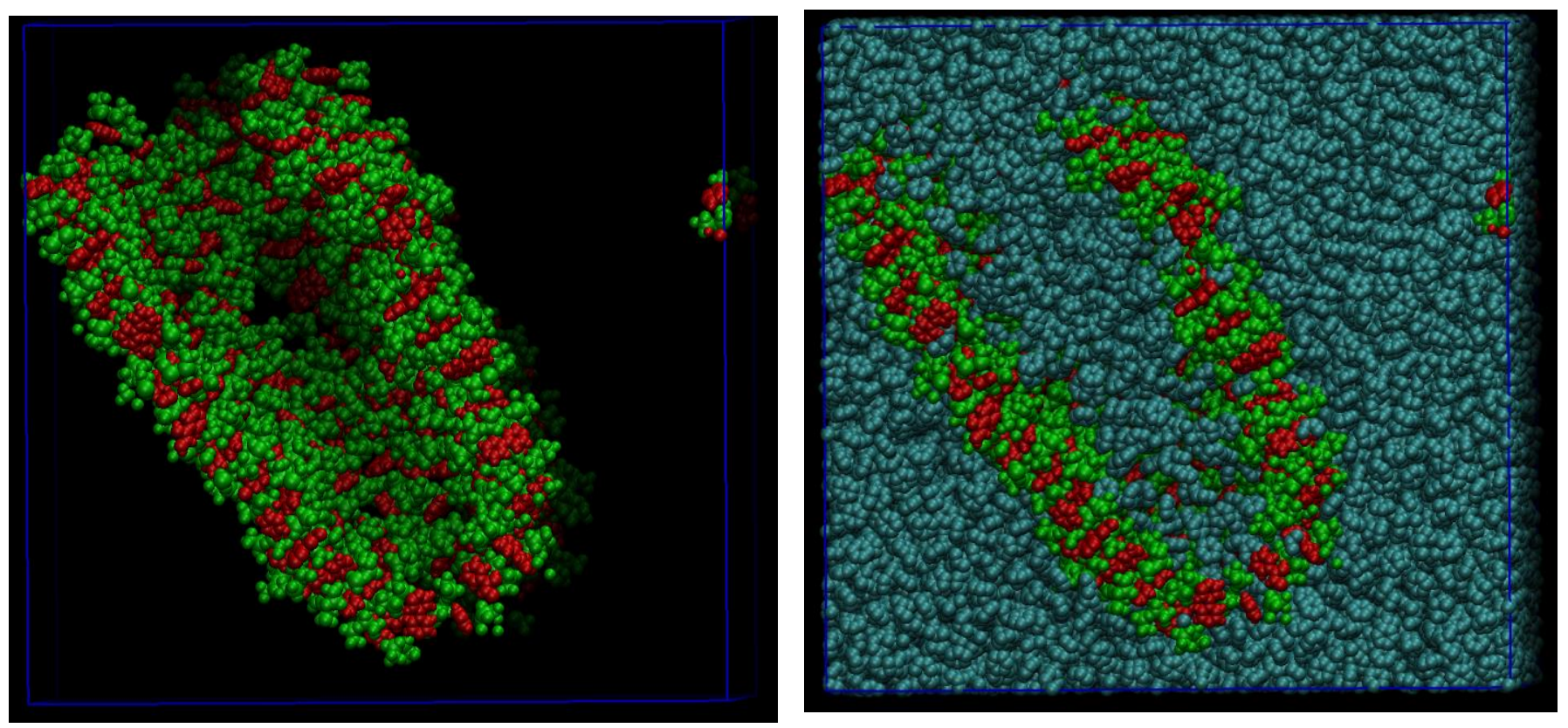

Figure S4: a snapshots of the $\mathrm{C}_{12} \mathrm{bpC}_{12}(1: 30)$ system. Pyridinium Ring (Red); $\mathrm{Tf}_{2} \mathrm{~N}$ (Green); Benzene (Cyan).
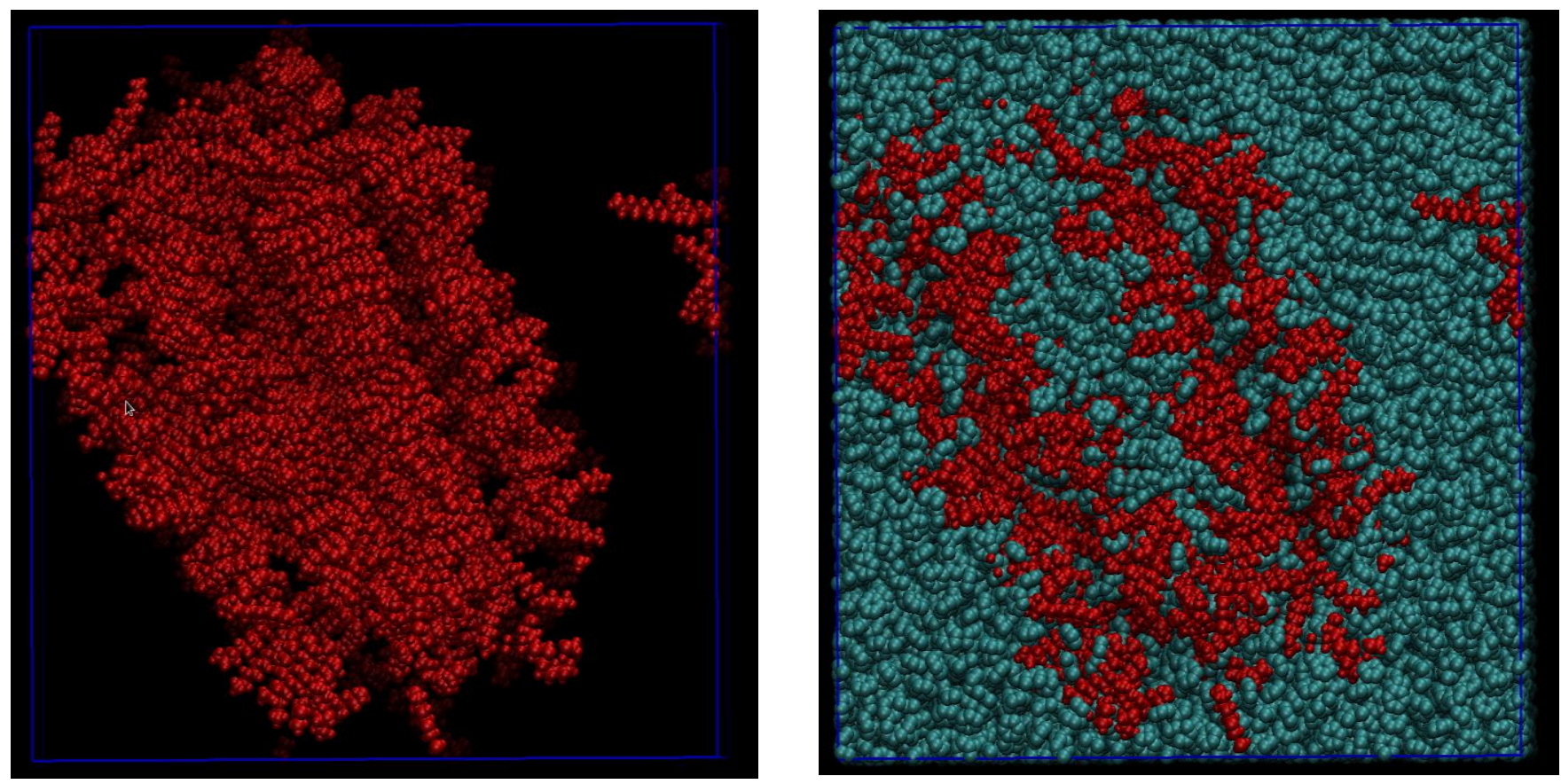

Figure S5: a snapshot (same as in Figure $\mathrm{S} 3$ ) of the $\mathrm{C}_{12} \mathrm{bpC}_{12}(1: 30)$ system. Side chains (Red); Benzene (Cyan). 

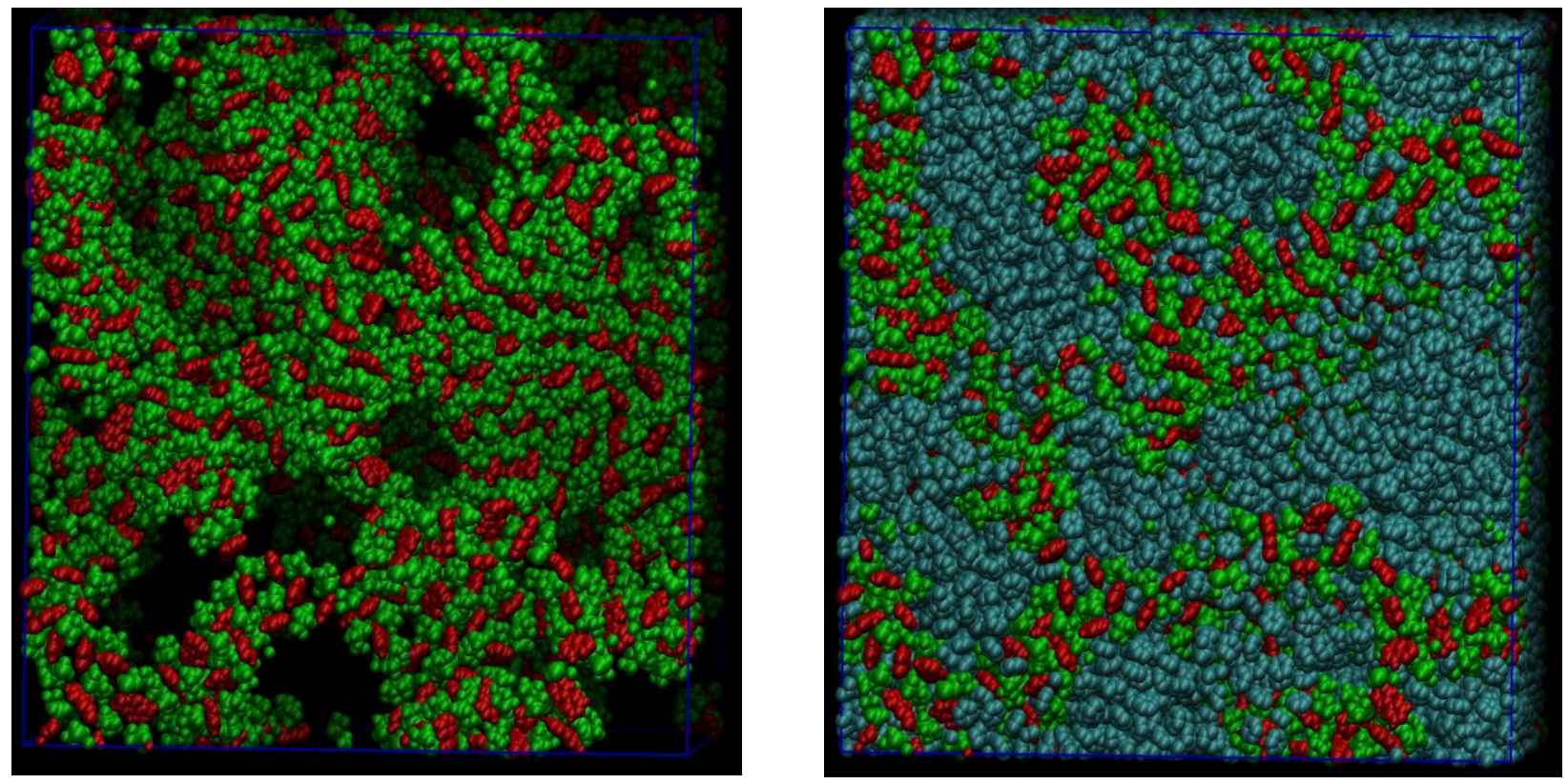

Figure S6: a snapshot of the $\mathrm{C}_{12} \mathrm{bpC}_{12}(1: 17)$ system. Pyridinium Ring (Red); $\mathrm{Tf}_{2} \mathrm{~N}$ (Green); Benzene (Cyan).

The coordination numbers between Benzene- $C_{\mathrm{n}}$ and Benzene- $N^{+}$

C12bpC12(1:17) C8bpC8(1:12)

$\mathrm{N}+0.153833 \quad \mathrm{~N}+0.177606$

C2 $\quad 0.199923 \quad$ C2 $\quad 0.219067$

$\begin{array}{llll}\text { C5 } & 0.271243 & \text { C3 } & 0.247103\end{array}$

C8 $\quad 0.303129 \quad$ C5 $\quad 0.29553$

$\begin{array}{llll}\mathrm{C} 10 & 0.337777 \quad \mathrm{C} 8 & 0.373285\end{array}$

C12 0.379189 


\section{Synthesis and characterization}

The compounds were synthesized according to the procedure reported in Refs [1-3].

1,1'-dinonyl-4,4'- bipyridinium di[bis(trifluoromethanesulfonyl)imide], CobpC9:

${ }^{1} \mathrm{HNMR}\left(500 \mathrm{MHz}, \mathrm{CD}_{3} \mathrm{OD}\right) \delta=9.27-9.25$ (m, 4H, Ar-H), 8.66-8.64 (m, 4H, Ar-H), 4.76-4.73 $\left(\mathrm{t}, J=7.6 \mathrm{~Hz}, 4 \mathrm{H}, \mathrm{N}-\mathrm{CH}_{2}-\right), 2.14-2.08\left(\mathrm{~m}, J=7.5 \mathrm{~Hz}, 4 \mathrm{H}, \mathrm{N}-\mathrm{CH}_{2}-\mathbf{C H}_{2}-\right), 1.44-1.32(\mathrm{~m}, 24 \mathrm{H})$, 0.93-0.90 (t, $\left.J=6.9 \mathrm{~Hz}, 6 \mathrm{H}, \mathrm{CH}_{3}\right) \mathrm{ppm} ;{ }^{13} \mathrm{CNMR}(125 \mathrm{MHz}) \delta=150.03,145.64,126.91$, 121.03, 118.49, (the quartet signal around 120 is related to CF3, $J=320.5$ ), 61.97, 31.56, 31.16, 29.02, 28.89, 28.72, 25.79, 22.28, 12.99 ppm; MS (ESI): positive: $m / z=205\left(\left[\mathrm{C}_{9} \mathrm{bpC}_{9}\right]^{2+}\right), 283$ $\left(\left[\mathrm{C}_{9} \mathrm{bp}\right]^{+}\right)$; negative: $\mathrm{m} / z=280\left(\mathrm{Tf}_{2} \mathrm{~N}\right)^{-}$.

1,1'-diundecyl-4,4'- bipyridinium di[bis(trifluoromethanesulfonyl)imide], $\mathbf{C}_{\mathbf{1 1}} \mathbf{b p C} \mathbf{C}_{\mathbf{1 1}}$ :

${ }^{1} \mathrm{HNMR}\left(500 \mathrm{MHz}, \mathrm{CD}_{3} \mathrm{OD}\right) \delta=9.25-9.24(\mathrm{~m}, 4 \mathrm{H}, \mathrm{Ar}-\mathrm{H}), 8.64-8.63(\mathrm{~m}, 4 \mathrm{H}, \mathrm{Ar}-\mathrm{H}), 4.76-4.73$ $\left(\mathrm{t}, J=7.6 \mathrm{~Hz}, 4 \mathrm{H}, \mathrm{N}-\mathrm{CH}_{2}-\right), 2.13-2.07$ (m, $J=7.5 \mathrm{~Hz}, 4 \mathrm{H}, \mathrm{N}-\mathrm{CH}_{2}-\mathbf{C H}_{2}$ ), $1.34-1.32$ (m, 32H), 0.93-0.90 (t, $\left.J=6.9 \mathrm{~Hz}, 6 \mathrm{H}, \mathrm{CH}_{3}\right) \mathrm{ppm} ;{ }^{13} \mathrm{C} \mathrm{NMR}\left(125 \mathrm{MHz}, \mathrm{CD}_{3} \mathrm{OD}\right) \delta=150.03,145.61$, $126.93,121.02$, 118.47, (the quartet signal around 120 is related to $\mathrm{CF} 3, J=320.5$ ), $61.98,31.65$, 31.14, 29.27, 29.22, 29.05, 29.02, 28.71, 25.79, 22.31, 13.02 ppm; MS (ESI): positive: $\mathrm{m} / z=233$ $\left(\left[\mathrm{C}_{11} \mathrm{bpC}_{11}\right]^{2+}\right), 311\left(\left[\mathrm{C}_{11} \mathrm{bp}\right]^{+}\right)$; negative: $m / z=280\left(\mathrm{Tf}_{2} \mathrm{~N}\right)^{-}$.

1,1'-didodecyl-4,4'- bipyridinium di[bis(trifluoromethanesulfonyl)imide], C12bpC12:

${ }^{1} \mathrm{HNMR}\left(500 \mathrm{MHz}, \mathrm{CD}_{3} \mathrm{OD}\right) \delta=9.25-9.23(\mathrm{~m}, 4 \mathrm{H}, \mathrm{Ar}-\mathrm{H}), 8.64-8.63(\mathrm{~m}, 4 \mathrm{H}, \mathrm{Ar}-\mathrm{H}), 4.76-4.73$ $\left(\mathrm{t}, J=7.6 \mathrm{~Hz}, 4 \mathrm{H}, \mathrm{N}-\mathrm{CH}_{2}-\right), 2.13-2.07\left(\mathrm{~m}, J=7.5 \mathrm{~Hz}, 4 \mathrm{H}, \mathrm{N}-\mathrm{CH}_{2}-\mathbf{C H}_{2}-\right), 1.44-1.31(\mathrm{~m}, 36 \mathrm{H})$, 0.93-0.90 (t, $\left.J=6.9 \mathrm{~Hz}, 6 \mathrm{H}, \mathrm{CH}_{3}\right) \mathrm{ppm} ;{ }^{13} \mathrm{CNMR}\left(125 \mathrm{MHz}, \mathrm{CD}_{3} \mathrm{OD}\right) \delta=149.91,145.61$, 126.93, (the quartet signal around 120 is related to CF3, $J=320.5), 61.90,31.66,31.15,29.31$, 29.31, 29.22, 29.05, 29.05, 28.71, 25.79, 22.32, 13.03 ppm; MS (ESI): positive: $\mathrm{m} / z=247$ $\left(\left[\mathrm{C}_{12} \mathrm{bpC}_{12}\right]^{2+}\right), 325\left(\left[\mathrm{C}_{12} \mathrm{bp}\right]^{+}\right)$; negative: $m / z=280\left(\mathrm{Tf}_{2} \mathrm{~N}\right)^{-}$.

1,1'-ditetradecyl-4,4'- bipyridinium di[bis(trifluoromethanesulfonyl)imide], $\mathbf{C}_{\mathbf{1 4}} \mathbf{b p C} \mathbf{1 4}$ :

${ }^{1} \mathrm{HNMR}$ (500 MHz, CD $3 \mathrm{OD}$ ): 9.26-9.25 (m, 4H, Ar-H), 8.65-8.64 (m, 4H, Ar-H), 4.76-4.73 (t, J $\left.=7.6 \mathrm{~Hz}, 4 \mathrm{H}, \mathrm{N}-\mathrm{CH}_{2}-\right), 2.14-2.08\left(\mathrm{~m}, J=7.5 \mathrm{~Hz}, 4 \mathrm{H}, \mathrm{N}-\mathrm{CH}_{2}-\mathbf{C H}_{2}-\right), 1.44-1.31$ (m, 44H), 0.93$0.90\left(\mathrm{t}, J=6.9 \mathrm{~Hz}, 6 \mathrm{H}, \mathrm{CH}_{3}\right) \mathrm{ppm} ;{ }^{13} \mathrm{CNMR}\left(125 \mathrm{MHz}, \mathrm{CD}_{3} \mathrm{OD}\right) \delta=150.02,145.63,126.91$, 121.03, 118.48, (the quartet signal around 120 is related to $\mathrm{CF} 3, J=320.5$ ), $61.97,31.66,31.16$, 29.38, 29.34, 29.34, 29.31, 29.22, 29.06, 29.06, 28.72, 25.80, 22.32, 13.02 ppm; MS (ESI): positive: $m / z=275\left(\left[\mathrm{C}_{14} \mathrm{bpC}_{14}\right]^{2+}\right), 353\left(\left[\mathrm{C}_{9} \mathrm{bp}\right]^{+}\right)$; negative: $m / z=280\left(\mathrm{Tf}_{2} \mathrm{~N}\right)^{-}$.

1-hexadecyl-1'-octyl-4,4'- bipyridinium di[bis(trifluoromethanesulfonyl)imide], C8bpC16:

${ }^{1} \mathrm{HNMR}\left(500 \mathrm{MHz}, \mathrm{CD}_{3} \mathrm{OD}\right) \delta=9.26-9.25$ (m, 4H, Ar-H), 8.65-8.64 (m, 4H, Ar-H), 4.76-4.73 (t, $\left.J=7.6 \mathrm{~Hz}, 4 \mathrm{H}, \mathrm{N}-\mathrm{CH}_{2}-\right), 2.14-2.08\left(\mathrm{~m}, J=7.5 \mathrm{~Hz}, 4 \mathrm{H}, \mathrm{N}-\mathrm{CH}_{2}-\mathbf{C H}_{2}-\right), 1.45-1.31(\mathrm{~m}, 36 \mathrm{H})$, $0.92-0.90$ (t, $\left.J=6.9 \mathrm{~Hz}, 6 \mathrm{H}, \mathrm{CH}_{3}\right) \mathrm{ppm} ;{ }^{13} \mathrm{CNMR}\left(125 \mathrm{MHz}, \mathrm{CD}_{3} \mathrm{OD}\right) \delta=150.03,145.63$, 126.91, 121.03, 118.48, (the quartet signal around 120 is related to CF3, $J=320.5$ ), 61.97, 31.16, 30.46, 30.67, 29.73, 29.37, 29.35, 29.31, 29.23, 29.07, 28.74, 28.68, 25.80, 22.32, 22.25, 13.03, 12.97 ppm; MS (ESI): positive: $m / z=247\left(\left[\mathrm{C}_{8} b_{p C C}\right]_{16}^{2+}\right), 381\left(\left[\mathrm{C}_{16} \mathrm{bp}\right]^{+}\right)$; negative: $m / z=280$ $\left(\mathrm{Tf}_{2} \mathrm{~N}\right)^{-}$. 


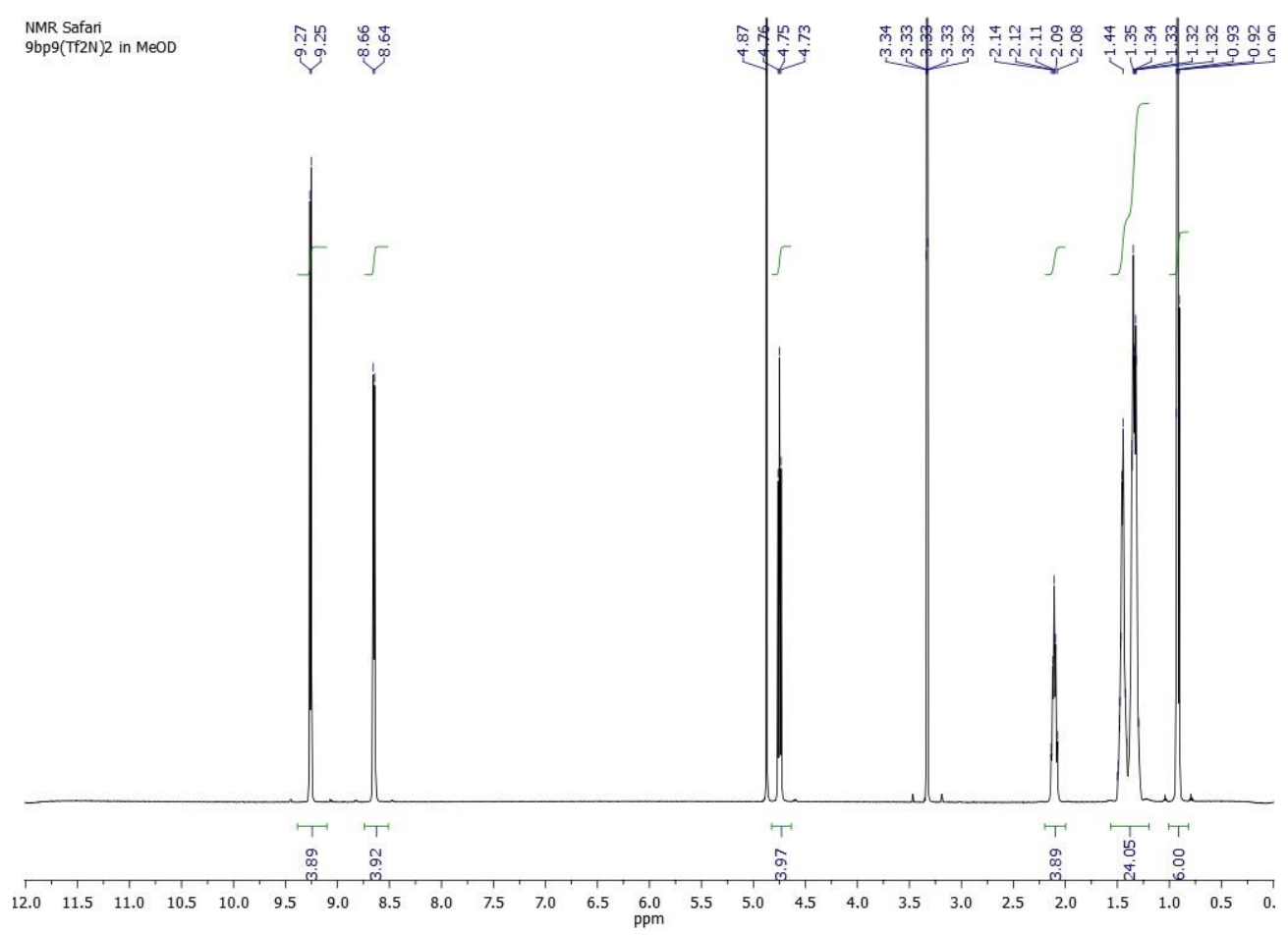

Figure S7. ${ }^{1} \mathrm{H}$ NMR spectrum of $\left[\mathrm{C}_{9} \mathrm{bpC} \mathrm{C}_{9}\right]\left[\mathrm{Tf}_{2} \mathrm{~N}\right]_{2}$.
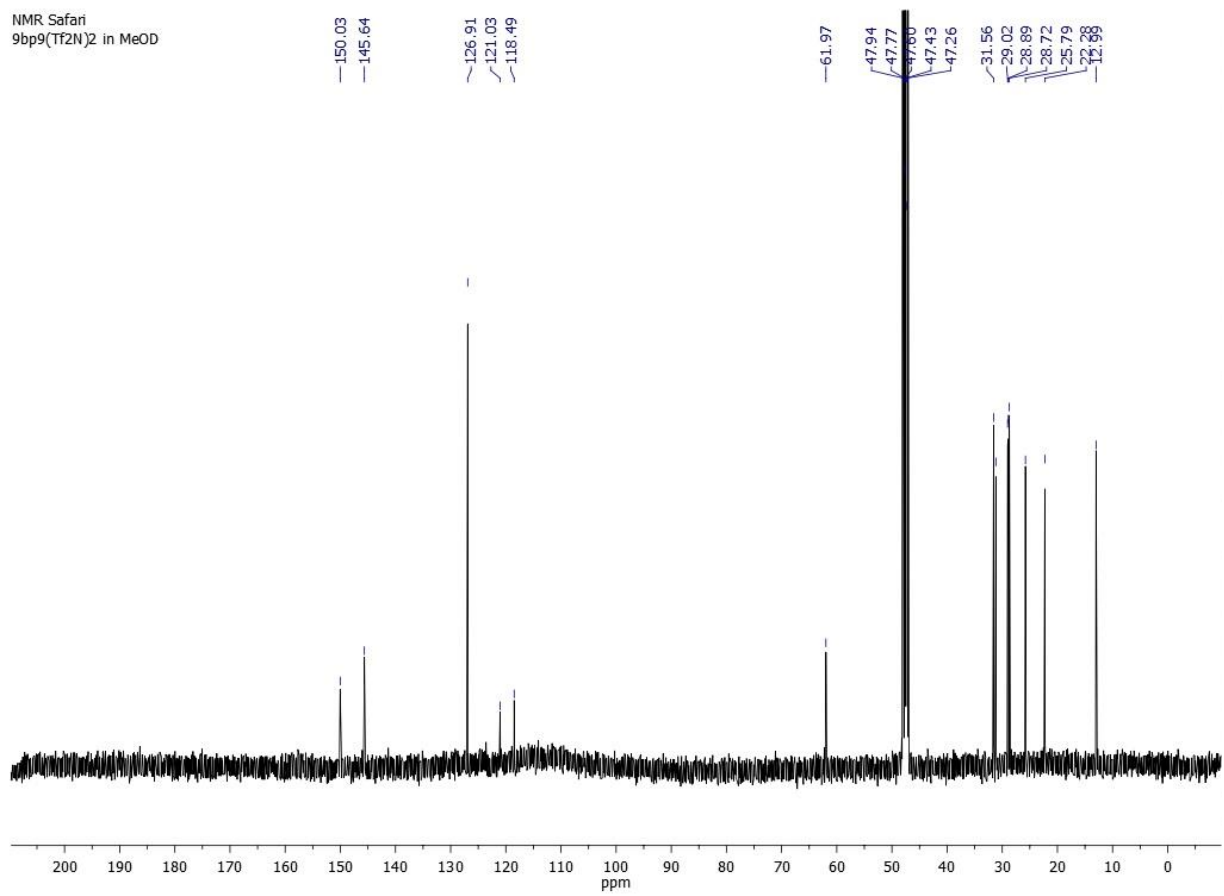

Figure S8. ${ }^{13} \mathrm{C}$ NMR spectrum of $\left[\mathrm{C}_{9} \mathrm{bpC} \mathrm{C}_{9}\right]\left[\mathrm{Tf}_{2} \mathrm{~N}\right]_{2}$. 


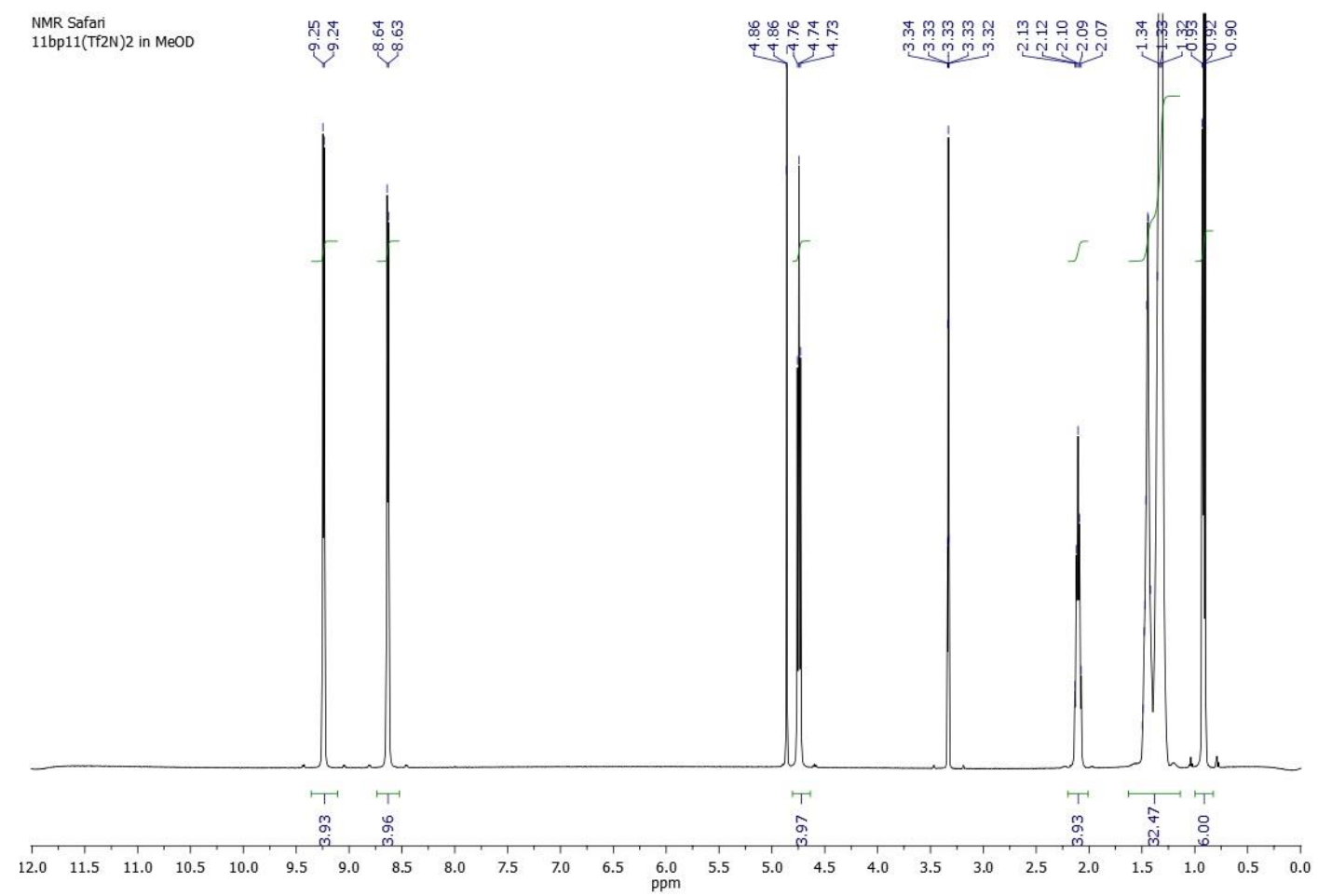

Figure S9. ${ }^{1} \mathrm{H}$ NMR spectrum of $\left[\mathrm{C}_{11} \mathrm{bpC}_{11}\right]\left[\mathrm{Tf}_{2} \mathrm{~N}\right]_{2}$.

NMR Safari

$11 \mathrm{bp} 11(\mathrm{Tf} 2 \mathrm{~N}) 2$ in MeOD

(1)
ले

i
๓ ผ ๓

-

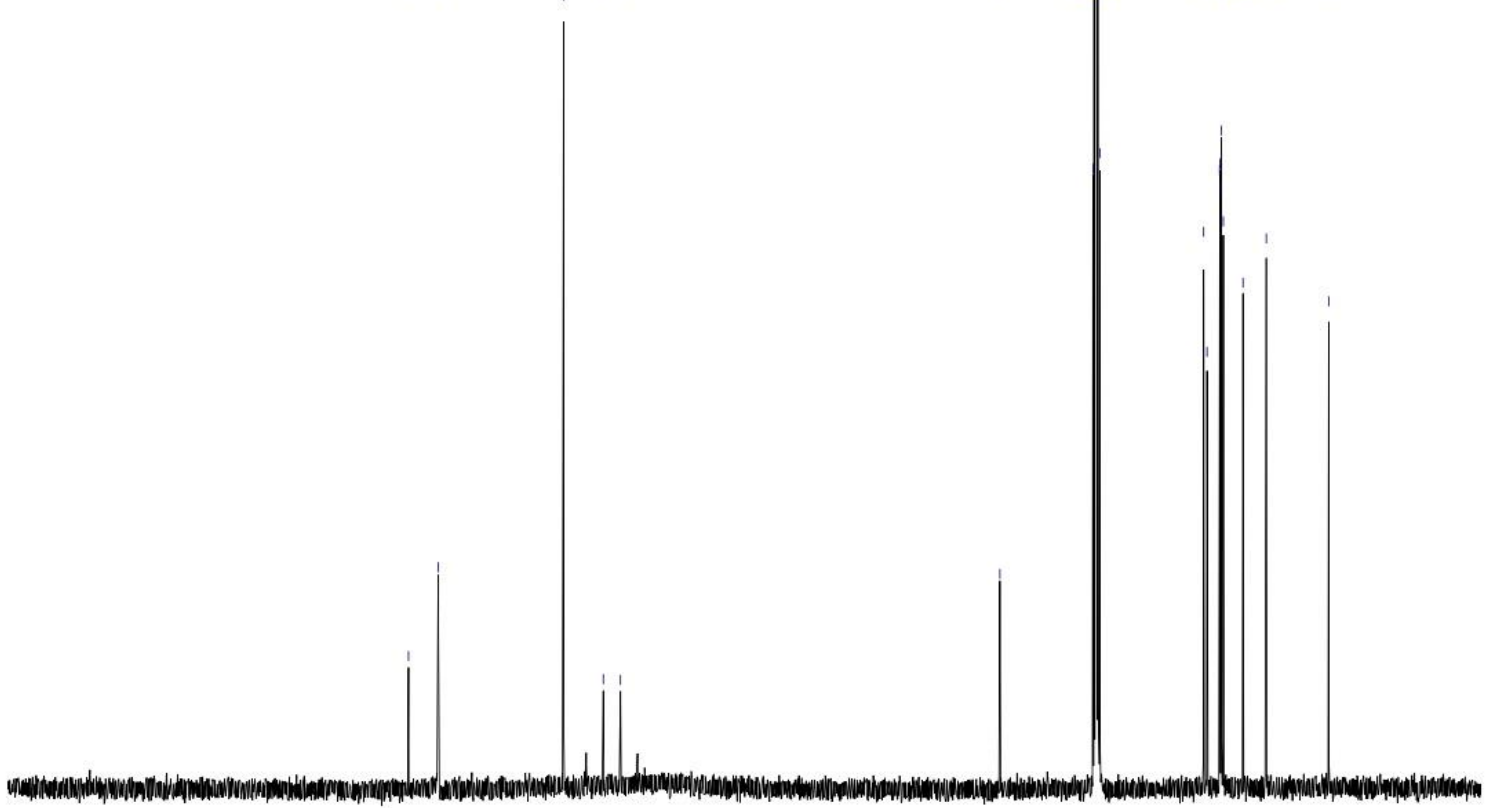

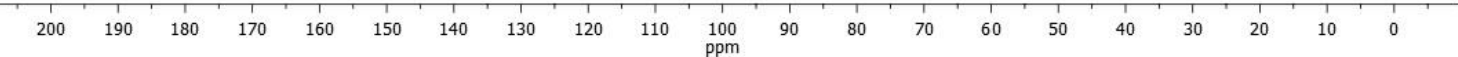

Figure S10. ${ }^{13} \mathrm{C}$ NMR spectrum of $\left[\mathrm{C}_{11} \mathrm{bpC}_{11}\right]\left[\mathrm{Tf} \mathrm{f}_{2} \mathrm{~N}\right]_{2}$. 


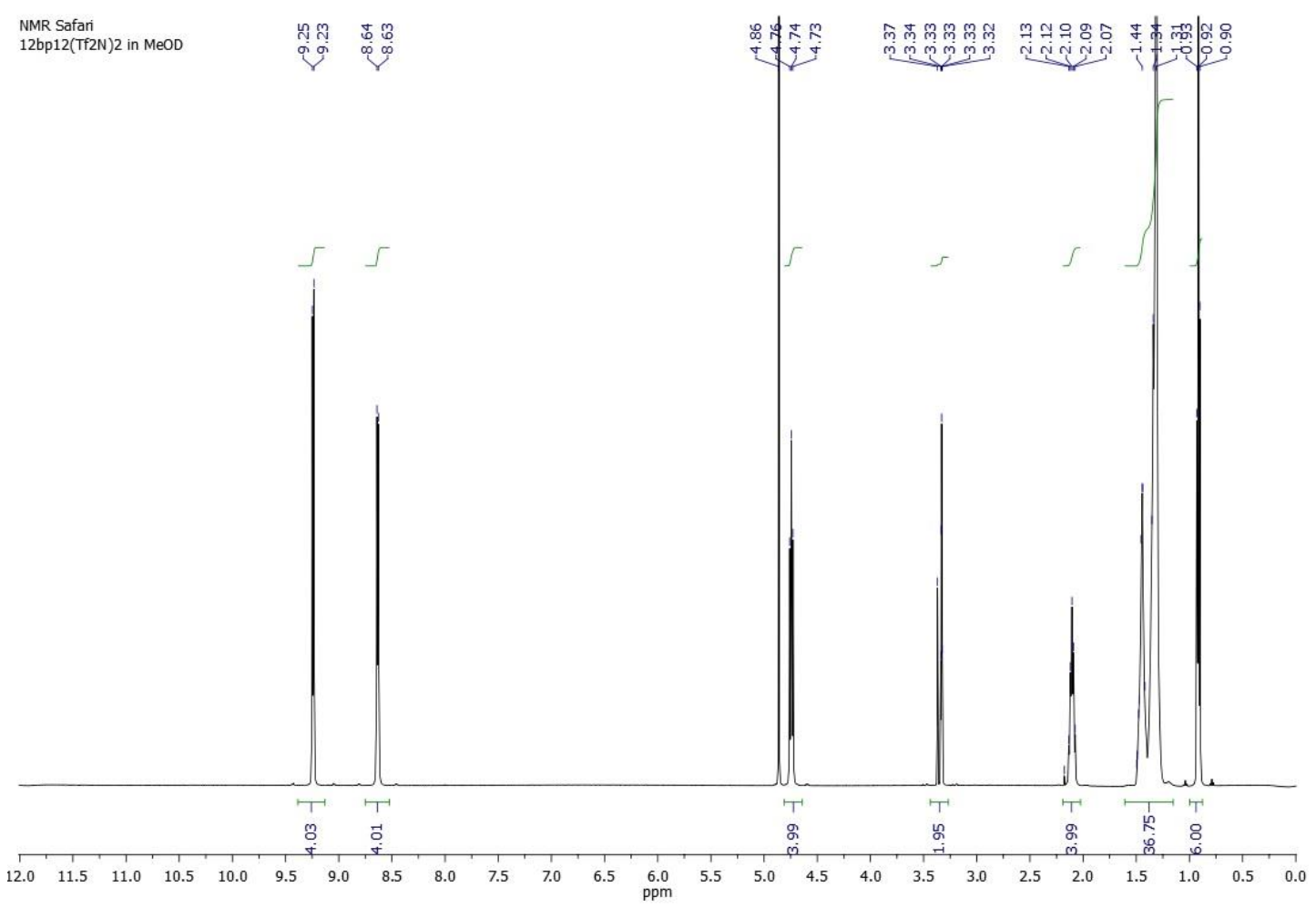

Figure S11. ${ }^{1} \mathrm{H} \mathrm{NMR}$ spectrum of $\left[\mathrm{C}_{12} \mathrm{bpC}_{12}\right]\left[\mathrm{Tf}_{2} \mathrm{~N}\right]_{2}$.

NMR Safari 12bp12(Tf2N)2 in MeOD

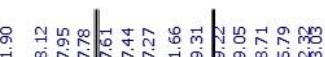

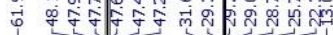

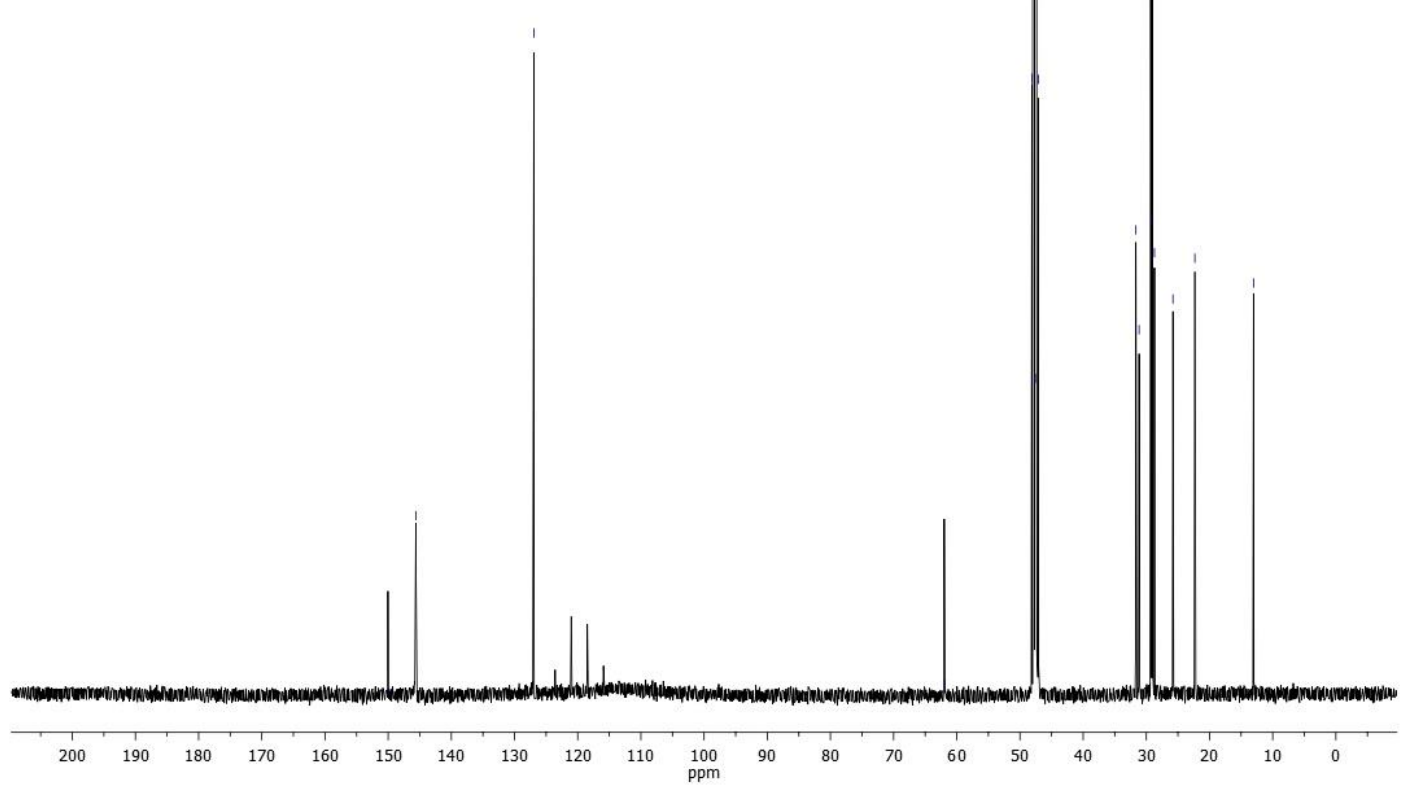

Figure S12. ${ }^{13} \mathrm{C}$ NMR spectrum of $\left[\mathrm{C}_{12} \mathrm{bpC}_{12}\right]\left[\mathrm{Tf}_{2} \mathrm{~N}\right]_{2}$. 


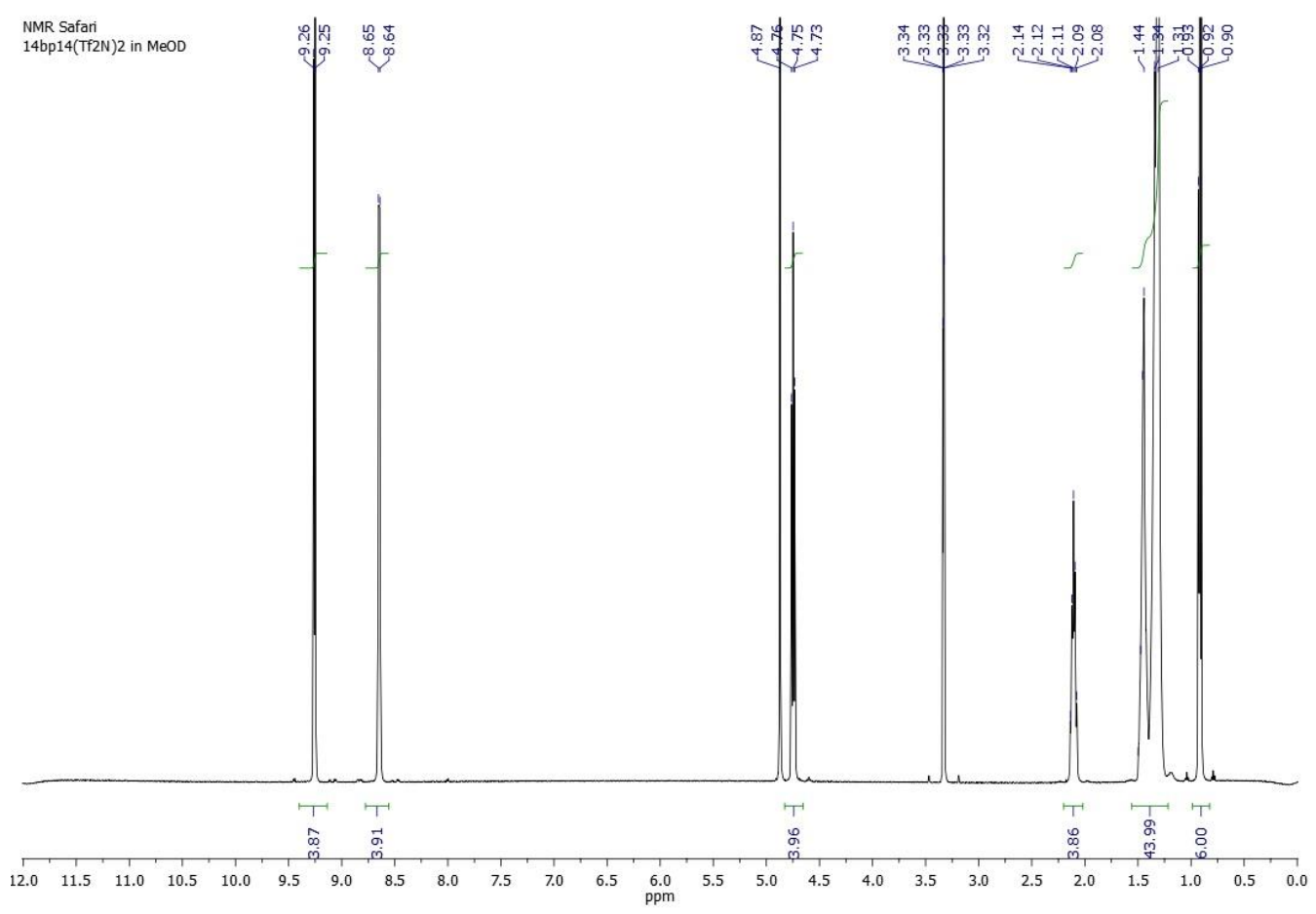

Figure S13. ${ }^{1} \mathrm{H}$ NMR spectrum of $\left[\mathrm{C}_{14} \mathrm{bpC}_{14}\right]\left[\mathrm{Tf}_{2} \mathrm{~N}\right]_{2}$.

NMR Safari

14bp14(Tf2N)2 in MeOD

웅

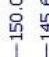

해에

군구

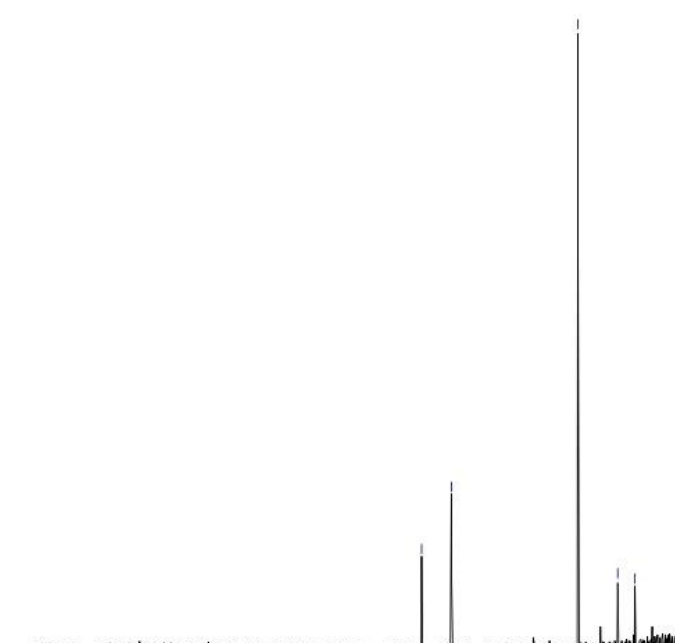

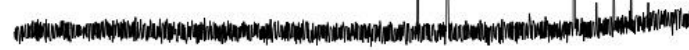

$\begin{array}{lllllllllll}200 & 190 & 180 & 170 & 160 & 150 & 140 & 130 & 120 & 110 & 1\end{array}$

Figure S14. ${ }^{13} \mathrm{C}$ NMR spectrum of $\left[\mathrm{C}_{14} \mathrm{bpC}_{14}\right]\left[\mathrm{Tf}_{2} \mathrm{~N}\right]_{2}$. 


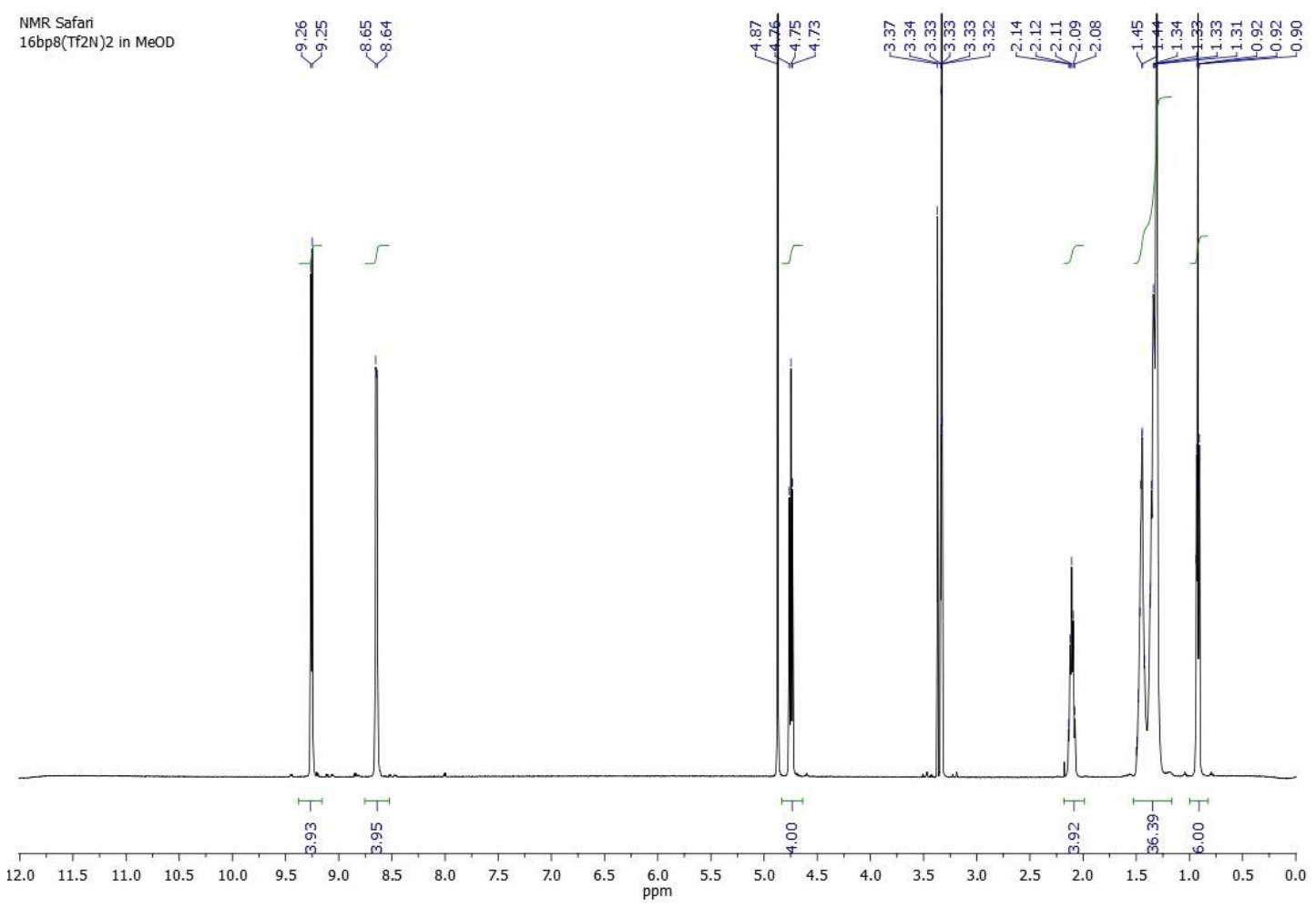

Figure S15. ${ }^{1} \mathrm{H}$ NMR spectrum of $\left[\mathrm{C}_{16} \mathrm{bpC} \mathrm{C}_{8}\right]\left[\mathrm{Tf}_{2} \mathrm{~N}\right]_{2}$.

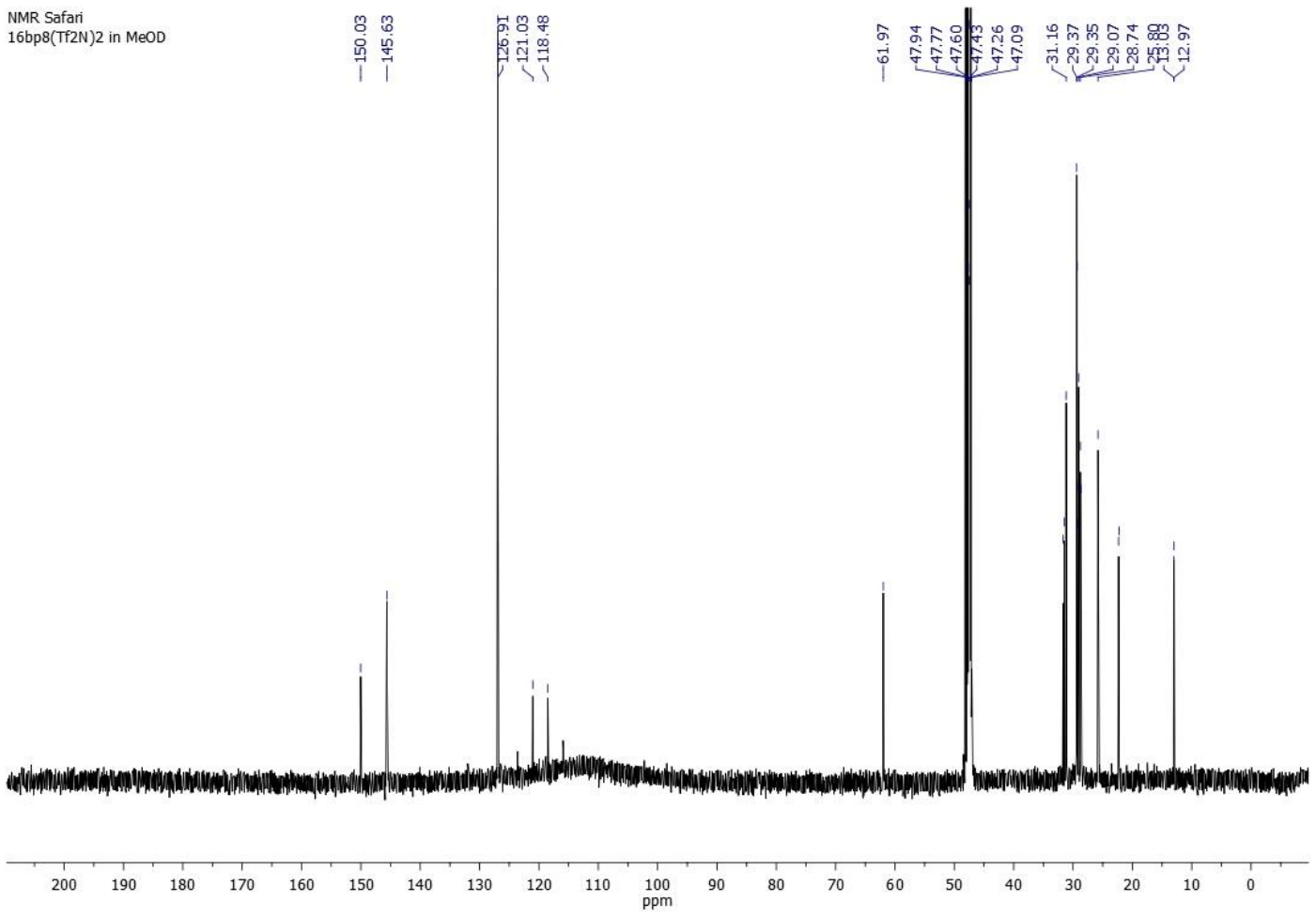

Figure S16. ${ }^{13} \mathrm{C}$ NMR spectrum of $\left[\mathrm{C}_{16} \mathrm{bpC}_{8}\right]\left[\mathrm{Tf}_{2} \mathrm{~N}\right]_{2}$. 


\section{References}

[1] S. Li, G. Saielli, Y. Wang, Phys. Chem. Chem. Phys. 2018, 20, 22730-22738.

[2] G. Casella, V. Causin, F. Rastrelli, G. Saielli, Liq. Cryst. 2016, 43, 1161-1173.

[3] G. Casella, V. Causin, F. Rastrelli, G. Saielli, Phys. Chem. Chem. Phys. 2014, 16, 5048-5051. 